\title{
Explanation of How to Run the Global Local Optimization Code (GLO) to Find Surface Heat Flux
}

\author{
Vivek Sahai \\ Salvador Aceves \\ Werner Stein
}

March 1999

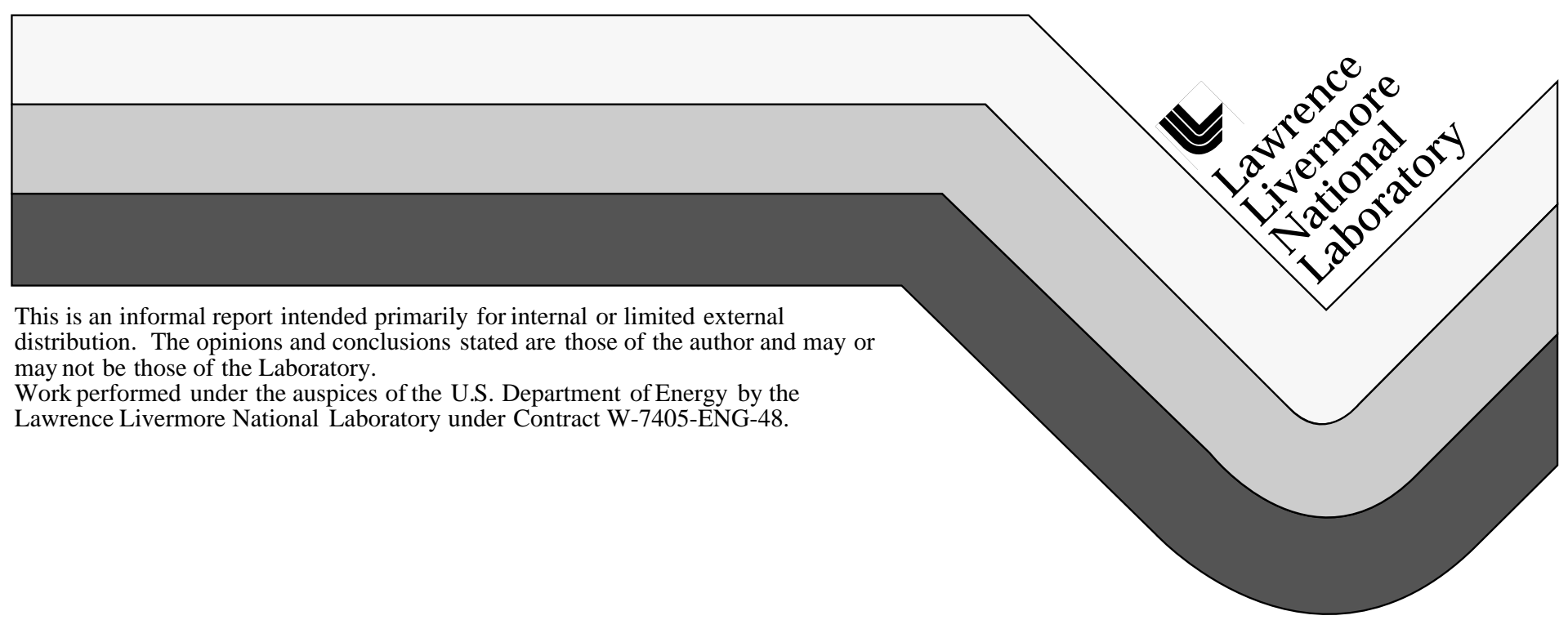




\section{DISCLAIMER}

This document was prepared as an account of work sponsored by an agency of the United States Government. Neither the United States Government nor the University of California nor any of their employees, makes any warranty, express or implied, or assumes any legal liability or responsibility for the accuracy, completeness, or usefulness of any information, apparatus, product, or process disclosed, or represents that its use would not infringe privately owned rights. Reference herein to any specific commercial product, process, or service by trade name, trademark, manufacturer, or otherwise, does not necessarily constitute or imply its endorsement, recommendation, or favoring by the United States Government or the University of California. The views and opinions of authors expressed herein do not necessarily state or reflect those of the United States Government or the University of California, and shall not be used for advertising or product endorsement purposes.

This report has been reproduced directly from the best available copy.

Available to DOE and DOE contractors from the Office of Scientific and Technical Information P.O. Box 62, Oak Ridge, TN 37831

Prices available from (423) 576-8401

Available to the public from the National Technical Information Service

U.S. Department of Commerce 5285 Port Royal Rd., Springfield, VA 22161 


\title{
Explanation of How to Run the \\ Global Local Optimization Code (GLO) \\ to Find Surface Heat Flux
}

by

\author{
Vivek Sahai \\ Salvador Aceves \\ Werner Stein
}
University of California
Lawrence Livermore National Laboratory
P.O. Box 808, L-140
Livermore, CA 94551




\section{Introduction}

From the evaluation[1] of the inverse techniques available, it was determined that the Global Local Optimization Code[2] can determine the surface heat flux using known experimental data at various points in the geometry. This code uses a whole domain approach in which an analysis code (such as TOPAZ2D or ABAQUS) can be run to get the appropriate data needed to minimize the heat flux function. This document is a compilation of our notes on how to run this code to find the surface heat flux. First, the code is described and the overall set-up procedure is reviewed. Then, creation of the configuration file is described. A specific configuration file is given with appropriate explanation. Using this information, the reader should be able to run GLO to find the surface heat flux.

\section{Description of GLO and Overall Setup Approach}

The GLO code [2] uses whole domain specification to find the surface heat fluxes Mathematically, whole domain specification[3-4]can be described as follows:

Find the Heat Flux Function E(q)

where $\mathrm{q}=\left\{\mathrm{q}_{1}, \mathrm{q}_{2}, \mathrm{q}_{\mathrm{i}}, \ldots . \mathrm{q}_{\text {nvariables }}\right\}$

such that $\mathrm{q}_{\text {minimum }}<\mathrm{q}_{\mathrm{i}}<\mathrm{q}_{\text {maximum }}$

where the values of $\mathrm{q}_{\mathrm{i}}$ minimize the figure of merit which is $\sum$ (Texperiment - Tcalc $)^{2}$

(the summation occurs over all time steps)

The optimization strategy that works best in GLO is the quasi-newton method with a finite difference gradient search technique. The iterative equations that describe the Newton's method are given below $(\mathrm{j}$ is the iteration number, $\mathrm{E}(\mathrm{q})$ is the heat flux function, and $\mathrm{q}$ represents the array of heat flux variables, and nvariables is the total number of variables):

$$
\begin{aligned}
& \nabla^{2} \mathrm{E}\left(\mathrm{q}_{\mathrm{j}}\right) \cdot \Delta \mathrm{q}=-\nabla \mathrm{E}\left(\mathrm{q}_{\mathrm{j}}\right) \\
& \mathrm{q}_{\mathrm{j}+1}=\mathrm{q}_{\mathrm{j}}+\Delta \mathrm{q}
\end{aligned}
$$

To set-up GLO to do the calculations, a configuration file is created. This file contains the commands to run the numerical analysis code(either TOPAZ2D or ABAQUS) to calculate the temperatures based on the current iteration values of the heat flux. Then ORION is used as a post-processing code that processes the t2plot file created by TOPAZ2D. ORION creates a file that contains the cooling curve data for the nodes that correspond to the actual experimental thermocouple positions. This configuration file also contains the details of the parameters that are to be optimized (heat flux variables). It sets the details of the iteration loop and how to calculate the figure of merit that the code uses to do the optimization.

Finally, the overall procedure used to get the heat flux results is described below :

(a) create sub-files (which are placed into the configuration file) containing the experimental thermocouple data,

(b) create the sub-file which runs the numerical analysis code,

(c) make the glo-configuration file,

(d) run the GLO analysis (for two iterations),

(e) check the *.log file to see if everything is working,

(f) run the GLO analysis, and finally,

(g) analyze the results by comparing with experimental data 
The key step in the procedure in running GLO is the creation of the configuration file. This file is described in the next section.

\section{GLO Configuration File (xxx.GCF)}

The configuration file is very important for running the GLO code. This file contains the following elements:

(a) GLO commands (such as glo-put, glo-get, loop, glo-file, and end glo-file)

(b) Analysis Code Run Commands (such as the TOPAZ2D/ABAQUS execution

command)

(c) Commands Used to Extract the Numerical Data (ORION execution command)

(d) Optimization Strategy ( Quasi-Newton technique)

(e) Optimization Parameters (The names of the heat flux variables, minimums, maximums)

(f) Analysis Code Input File (TOPAZ2D input file/ ABAQUS input file)

(g) Figure of Merit Calculations

(h) Command File listing ORION commands to print out time vs. temperature data from the $\underline{t} 2$ plot file created by the TOPAZ2D code (ORION Command File)

(i) Experimental Data used in the Figure of Merit Calculation.

The configuration file consists of several sections(sub-files) which contain the above elements. Each section begins with the command "glo-file = name of the file" and ends with the command "end-glo file".

The first section is called the "iloc" sub-file. This sub-file contains the information of where the GLO executable file resides, the iteration analysis loop, optimization strategy, and the heat flux curve parameter names with their minimum, maximum, and initial values (elements (a) through (e) are placed here). The iteration loop lets the GLO code place the values of the heat flux in the numerical analysis code input file, run the post-processor, and then call the file to do the figure of merit calculation (done with the glo-get command).

The second section is a slightly modified form of the numerical analysis code input file. This sub-file contains the details of the numerical analysis options, material properties, mesh, node numbers and locations, element definitions, heat flux boundary condition location, and heat flux curve details. In this sub-file, a glo-format command is used in the details of the heat flux curve which tells GLO where to put the values of the heat flux needed as a boundary condition to run the analysis code(element (f)).

The third section is the actual figure of merit calculation. This sub-file is given the name, calc. In this sub-file, the variables are defined and the names of the files are given from which to read both the experimental and numerical values. Then a do loop calculates the figure of merit and finally the value is printed out (element $(\mathrm{g})$ ).

The fourth section is the actual command sequence to print out the numerical values from the analysis code (element (h)). Finally, the last set of sub-files contain the experimental thermocouple data (one set of time vs. temperature data in each file)(element i). The specific glo-commands used in these files are further described in the GLO manual[2]. The best way to understand the configuration file is through the example given in the last section of this document.

When creating the GLO configuration file, the following points must be kept in consideration: 
I. Make sure the number of significant figures is enough for the finite difference calculations; if not, change the value of the dx_grad parameter. In our work, to determine the surface heat fluxes of the Saturn Gear Blank, we recommend dx_grad be set to 0.001 .

II. In the figure of merit calculations (glo-file named calc), make sure the number of points in the experimental data equals the number of points generated by the postprocessing code, ORION. To avoid the need for interpolation, make sure the comparison between the numerical and experimental data occur at the same time step.

In the example section, a specific GLO gcf file is given which will emphasize the information given above. To understand more detail of what each command in GLO does, please refer to the GLO users manual[2].

\section{Running the GLO code and the Files Created During the Run}

Once the gcf file is created, the command line to run GLO on a SGI IRIX type system is simply the following:

$$
\text { glo xxx.gcf > out \& }
$$

where xxx.gcf is the name of the gcf file, and out is the file where the screen output is directed to. After hitting the return key to start the run, a number(nnnn) is given which is the process id of the GLO calculations, If one needs to kill the calculations, use the following command:

kill -9 nnnn

During the run, the following files are created which give the status of the run:

(a) out which contains the screen output (from TOPAZ2D and ORION for example and information from the GLO controller with the figure of merit)

(b) xxx.log which contains the progress of GLO along with any error messages or other troubles during the run along with the commands performed during the run for each iteration

(c) local.11 which documents the values of the variables of each run and the commands from the loop executed during each run, the type of run (i.e. gradient evaluation or newton step) and list the figure of merit during the run.

(d) local.u1 which lists the iteration number and value of the figure of merit

(e) varvals which contains the current values of the parameters to be optimized (ie. heat flux values), and finally,

(f) glo-nnnn.recover which is used to restart GLO if the system crashes during a run.

To use the recover file, be sure not to make any changes to the gcf file and use the command:

glo -recover glo-nnnn.recover xxx.gcf > out \&

During the GLO run, each iteration's work is stored in a separate directory, i.e. ./pyyyy/ 
where yyyy is the iteration number. This directory contains the analysis code input file with the current values of heat flux for that iteration, and all the command files used to extract the numerical output, as well as the numerical output, etc. To save disk space, one can use "rm" commands in the gcf to delete unnecessary files. It is strongly recommended that after the run, all these directories be removed except for the last iteration number directory!

\section{Files to Examine After the GLO Run}

The values of all the heat flux parameters are contained in the solution.0000 and varvals files. In the solution.0000 file, the final value of the figure of merit and the last iteration number is also given. The local.u2 file contains the values of the figure of merit for each iteration. To understand the solution, one should examine the last iteration number directory. The final version of the analysis code input file is contained in the $. / \mathbf{p x x x} /$ directory where $\mathrm{xxxx}$ is the number of the last iteration. One should run this file to compare the results of the predicted temperatures with the experimental data. Examples of how these files look like are given in the example problem in the next section. Again for space management, one can delete all the files in the ./pyyyy/ directories before the last iteration XXXX.

\section{Example Problem}

A two-dimensional square cavity 10 units long by 10 units high has a uniform constant heat flux applied to the back face which causes a rise in temperature from a 0 degrees uniform initial temperature. Known temperature data exists for the mid-point position on the back face. The other three faces are assumed to be insulated. The objective is to use GLO to find the value of the heat flux.

The diagram of the situation is shown below:

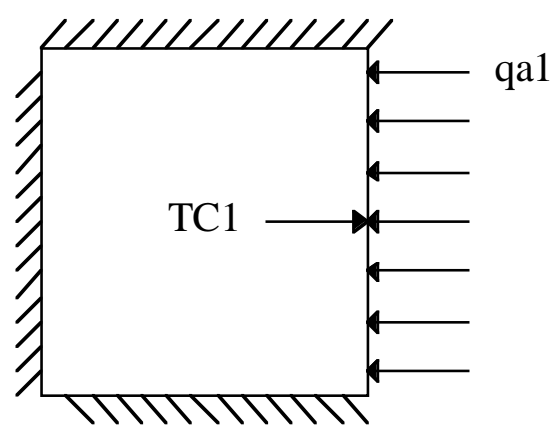

Figure 1. Sketch of Square Cavity with a Uniform Heat Flux applied on the back surface and known temperature data located at the mid-point on the back surface.

An outline of the calculational procedure that GLO uses is given in Figure 2. The file names on the left are the ones that are created by GLO from the information in the gcf file. The file names below each box are the ones created by the code. The "fom" stands for the figure of merit variable calculated by the calc file. This value is used by the optimizer to determine whether to continue iterating or print out the results. The variable name "qa1" is the value of the heat flux parameter which is specified in the analysis code input file (ttest1.ref) that is being optimized by GLO. The value of the heat flux to be found is given the variable name qa1. As seen from the outline, first GLO reads the entire gcf file, then 
puts the initial value of "qa1" in the file ttest1.ref at the position indicated by the glo-format comand, and creates the file ttest1. Then ttest 1 is used in TOPAZ to do the transient

Outline for GLO Calculations

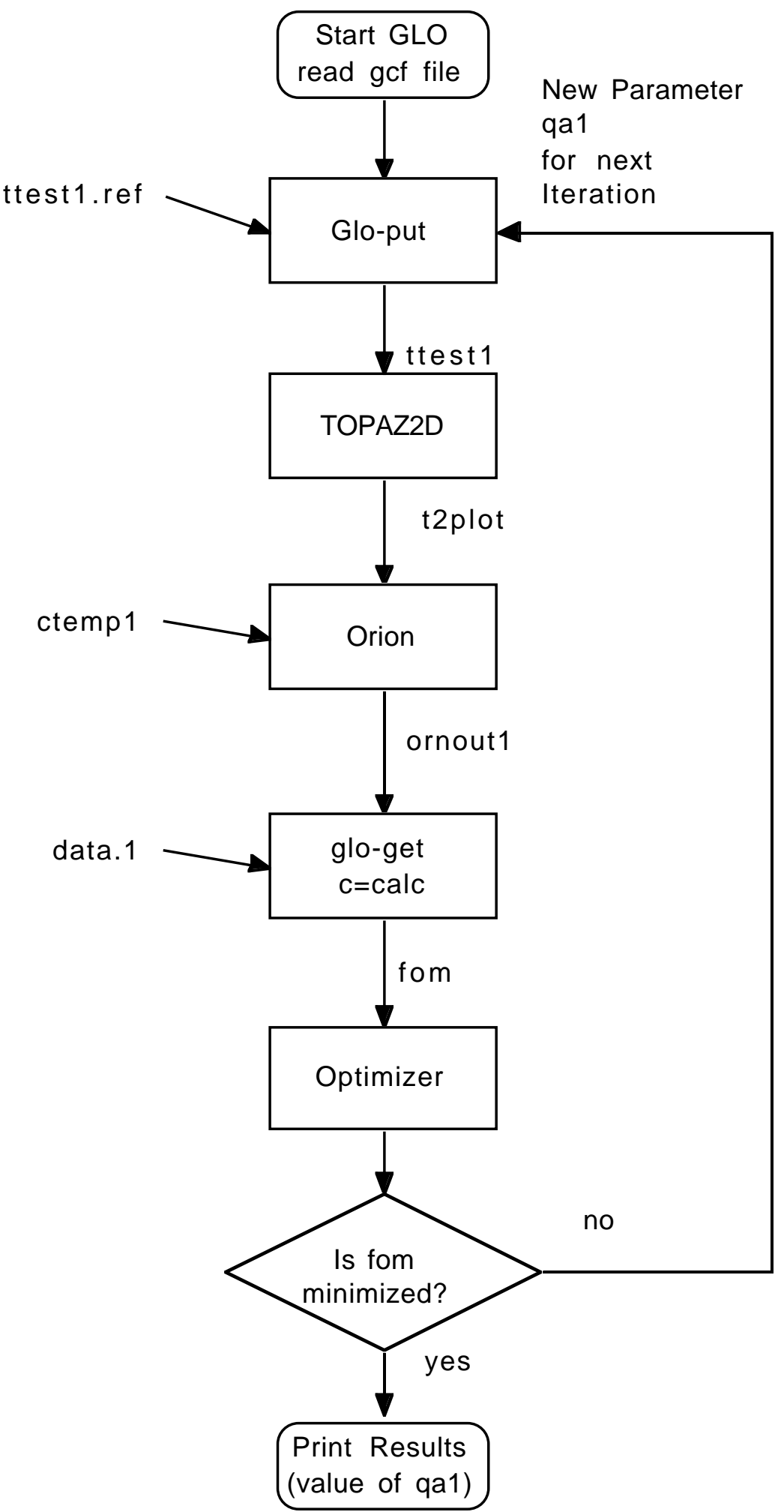


Figure 2. Flow Chart Showing Iteration Loop of GLO Calculations

heat transfer analysis and the results from the TOPAZ analysis are placed in the output file t2plot. Then ORION is used to get the cooling curve data at the node corresponding to the location of TC1. The commands to get this information are contained in the sub-file in the gcf named ctemp1. ORION puts this information in the file ornout which is moved to ornout1. Then this information from ornout 1 and the experimental data data. 1 is read into the sub-file named calc which determines the figure of merit whose value is stored in the variable named "fom". This value is used in the optimizer which then determines the next value of qa1 for the next iteration. If the figure of merit is optimized, then the results are printed in the names of the files listed in the previous section.

The GLO configuration file used to solve this problem using the heat transfer analysis code TOPAZ2D and the post-processing code ORION is given in Table 1. There are total of five sub-files or sections that make up this gcf. Please look at the \# or $\$$ comment cards which explain what each line does. The words in bold face give specific details of each section or line of the configuration file.

The resulting files created by GLO (i.e. local.11, local.12, local.u2, and solution.0000) are given in the Appendix. The results show that a total of five iterations were performed. The first iteration was an initial evaluation followed by 2 Newton steps and 2 gradient evaluations. The values of the figure of merit was quickly minized to value less than 1 . The final value of the optimized heat flux was 0.09975 .

To analyze the results, the GLO calculated heat flux value can be checked by doing a direct simulation(i.e. a TOPAZ2D transient heat conduction simulation with the GLO predicted value of heat flux used as a boundary condition). The comparison between the calculated temperatures(using the optimized value of qa1) and the experimental data is shown in Figure 3. As one can see, the agreement between the GLO results and the known data is very good.

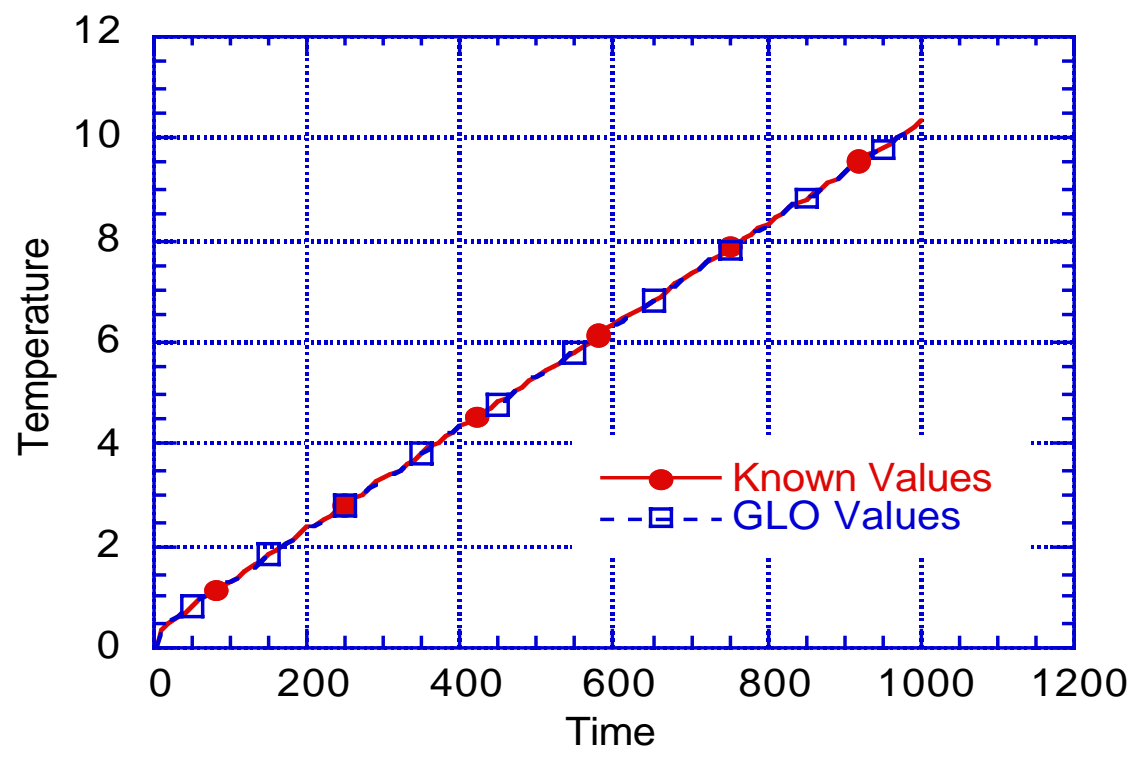

Figure 3. GLO calculated Temperatures vs. Known Data 


\section{Summary}

This document has given the reader the necessary information to set-up the GLO code to perform whole domain optimization to find the surface heat flux. Our work with GLO has shown the usefulness of this approach. For example, previous work at LLNL has shown the ability of the code not only predict the surface heat flux but the variation of the heat flux along the surface[1]. The ability of GLO to calculate surface heat fluxes for a horizontally quenched Saturn Gear Blank in non-agitated oil[5] has been demonstrated. is as follows:

In conclusion, a summary of the procedure for setting up and using the GLO code

(a) set up the experimental data files

(b) set up the numerical analysis input file

(c) use this information in the creation of a GLO configuration file

(d) run GLO

(e) analyze the results.

\section{References}

[1] M.Y. Lin, M. Murphy, A. Shapiro, W. Stein, "Numerical Methods for Inverse Heat Transfer Analysis”, Lawrence Livermore National Laboratory Report, September 1997.

[2] R. Matzke, “GLO User's Manual”, Lawrence Livermore National Laboratory, 1995.

[3] G.S. Dulikravich, T.J. Martin, "Inverse Shape and Boundary Condition Problems and Optimization in Heat Conduction", in Advances in Numerical Heat Transfer Volume 1 editors W. J. Minkowycz and E.M. Sparrow, Talyor and Francis, Washington DC 1997 pg. 409.

[4] J.E. Dennis Jr., Numerical Methods for Unconstrained Optimization and Nonlinear Equations, Prentice Hall, Inc. NJ, 1983.

[5] V. Sahai, Analysis of an Oil Quenched Gear Blank, Presentation at First Components Demonstration Meeting, Kissimmee, Florida February 23-25, 1998. 


\section{Table 1. GLO Configuration File(square.gcf) used to Solve Square Cavity Problem}

\# A \# comments out a whole line

\# A $\$$ comments till the end of the line

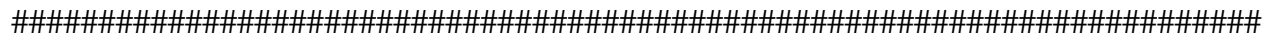

\# square.gcf \$ name of this gcf file

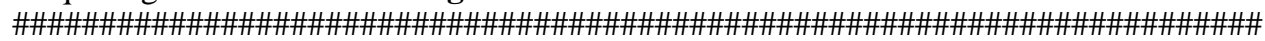

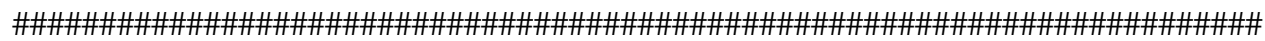
optimizer = /usr/people/sahai/GLO/bin/local i=iloc \$location of glo-excutable and

\# name of first sub-file which contains main loop

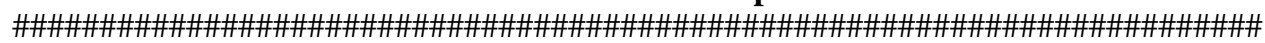

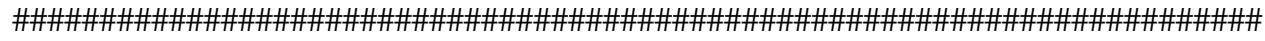
$\mathrm{ncpu}=1 \quad$ Max number of cpus available

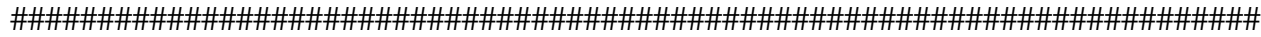

\# Section Number 1 (sub-file named iloc)

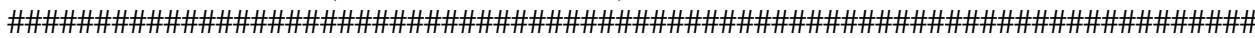

glo-file $=$ iloc

\$ initialize the non-default optimization code settings

settings

max_pfn 0102 \$ run loop this many times

parallel $1 \quad \$$ run one problem at a time in parallel

dx_grad 0.001 \$ relative finite difference gradient step

end settings

\# define the local optimization problem analysis loop

loop $\$$ this starts the loop (outline given in Figure 2)

glo-put $\mathrm{i}=$ ttest 1 .ref $\mathrm{o}=$ ttest 1

\# the above command takes the sub-file ttest1.ref(the input file that is specified given

\# in the sub-file named ttest1.ref within this gcf file) places the value of the heat flux

\# variable qa1 into ttest1 and produces the input file, ttest1, for the TOPAZ2D

\# analysis. Each iteration produces a ttest1 file that always contains the latest value

\# of qa1

topaz2d.sgi i=ttest1 \$ execution line for TOPAZ2D

\# TOPAZ2D produces plot files that contain the calculational output

\# The name of this file is t2plot

orion.sgi $\mathrm{g}=\mathrm{t} 2$ plot $\mathrm{c}=\mathrm{ctemp} 1$ \&exution line for orion to post-process results

\# ctemp1 is a sub-file given in this gcf. ctemp1 contains commands that create the

\# time temperature data for the node at the location of TC1 (see Figure 1) This data is

\# stored in the file name ornout

mv ornout ornout1 \$ moves analysis results to a file named ornout1 to be read by calc

rm dig.ps*

$\$$ unix commands to remove unneccessary files

rm t2plot

rm tprint

rm t2dump*

rm newfile

glo-get $\mathrm{c}=$ calc

\# name of glo sub-file (given in this gcf) which does objective function analysis

end loop $\$$ used to end the optimization analysis loop

\$ define the optimization strategy

strategy

local nlqpeb \$ for Quasi-Newton with finite difference gradient

\#

\# Heat Flux Parameter to be optimized is defined here.

\# parameter name, initial value, lowerbound, upperbound, 0.0

\# 
parameter qa1 $\quad 0.5 \mathrm{e} 0 \quad 0.0001 \quad 1.0 \mathrm{e} 3 \quad 0.0$

\# qa1 represents the name of the value of the unknown heat flux that has to be \# optimized. This parameter is placed in sub-file named ttest1.ref.

\# This line specifies, for qa1, the initial value 0.5 , minimum value 0.0001 and \# maximum value 1.0e3. For this type of optimization procedure each of these \# parameter lines must end in 0.0

end strategy \$after all parameters are named use this command to end the \# optimization strategy description

run

\# always use the run command to end the iloc file so that glo will start running! end glo-file

\# always use glo-file = name to begin a file and end glo-file to end a file

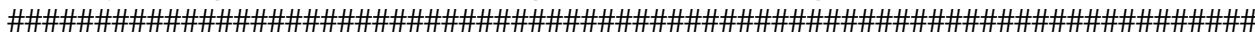
\# Section Number 2 - TOPAZ2D input file (sub-file named ttest1.ref)

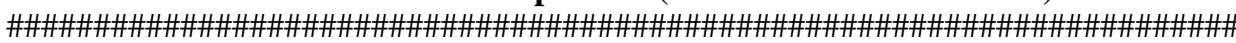
glo-file = ttest 1. ref

* This is a TOPAZ2D input file and therfore in this file every line
* beginning with a * represents a comment line

* TOPAZ2D Problem Definition: Card 1

* Field

* [1] Title

topaz input file: square problem for glo run

*

* TOPAZ2D Problem Definition: Card 2

* Field

Field

* [1] Number of Materials

[ 7] Number of data pairs

* [2] Number of nodes

* [3] Number of elements

* [4] Type of geometry

* [5] Bandwidth minimization

[ 8] Number of slidelines

[ 9] Total number of slave nodes

[10] Total number of master nodes

[11] Solution Method

[6] Number of function curves[12] Conjugate gradient convergence tolerance

* TOPAZ2D Problem Definition: Card 3

* Field Field

* [1] Number of elements with [ 6] Number of radiation internal heat generation boundary conditions

[2] Number of nodes with non-zero initial temp.

[ 7] Number of enclosure radiation segments

[3] Number of nodes with [ 8] Number of radiation bands temp. boundary conditions [ 9] Number of emissivity vs. wavelength curves

* 4] Number of flux boundary [10] Radiation calculation type condition segments [11] Number of special internal boundary elements

[5] Number of convection boundary condition segments

[12] Number of bulk nodes

[13] Number of bulk node segments

[14] Number of chemical reaction elements

[15] Number of fluid flow elements

$\star$

* TOPAZ2D Problem Definition:

* Field

* [1] Analysis type

* [2] Time step code

* [3] Node and element dump step interval for printing

* [4] Node and element dump

$\begin{array}{lllllllll}0 & 0 & 0 & 0 & 0 & 0 & 0 & 0 & 0\end{array}$

Card 4

Field

[5] Node and element dump time interval for printing

[6] Node and element dump time for post-processing

[7] Number of steps between restart dumps 


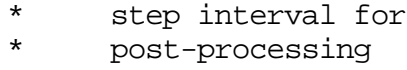

NOTICE: we are using

[5] Maximum time step size

[6] Maximum temperature change in each time step above which time step will be decreased

[7] Time step control parameter

* Therefore, the total number of datapoints will be 101. $0.0000 \mathrm{E}+00 \quad 1000.0 \quad 1.0$

* TOPAZ2D Problem Definition: Card 6

* Field

Field

* [1] Problem type

* [2] Maximum number of

* $\quad$ stiffness matrix

* reformations per time

* step

0

0.0

every 10 steps!

$\begin{array}{lll}0 & 1 & 100.0000 E+001.0000 E+00\end{array}$

*material name, number, and properties

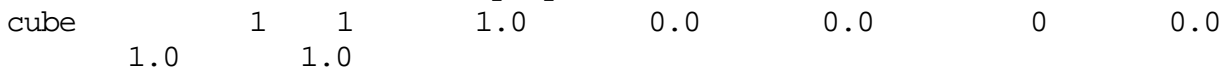

* node number increment $\mathrm{x}$ and $\mathrm{y}$ location

$\begin{array}{llll}1 & 0.0 & 0.000000 & 0.000000\end{array}$

$0.01 .000000 \quad 0.000000$

$0.02 .000000 \quad 0.000000$

$0.03 .000000 \quad 0.000000$

$0.0 \quad 4.000000 \quad 0.000000$

$\begin{array}{llll}0.0 & 5.000000 & 0.000000\end{array}$

$\begin{array}{llll}0.0 & 6.000000 & 0.000000\end{array}$

$\begin{array}{lll}0.0 & 7.000000 & 0.000000\end{array}$

$\begin{array}{lll}0.0 & 8.000000 & 0.000000\end{array}$

$\begin{array}{lll}0.0 & 9.000000 & 0.000000\end{array}$

$\begin{array}{lll}0.0 & 10.000000 & 0.000000\end{array}$

$\begin{array}{lll}0.0 & 0.000000 & 1.000000\end{array}$

$\begin{array}{lll}0.0 & 1.000000 & 1.000000\end{array}$

$0.0 \quad 2.000000 \quad 1.000000$

$\begin{array}{lll}0.0 & 3.000000 & 1.000000\end{array}$

$\begin{array}{lll}0.0 & 4.000000 & 1.000000\end{array}$

$0.0 \quad 5.000000 \quad 1.000000$

$\begin{array}{lll}0.0 & 6.000000 & 1.000000\end{array}$

$\begin{array}{lll}0.0 & 6.999999 & 1.000000\end{array}$

$\begin{array}{lll}0.0 & 8.000000 & 1.000000\end{array}$

$\begin{array}{lll}0.0 & 8.999999 & 1.000000\end{array}$

$0.010 .000000 \quad 1.000000$

$\begin{array}{lll}0.0 & 0.000000 & 2.000000\end{array}$

$0.0 \quad 1.000000 \quad 2.000000$

$\begin{array}{lll}0.0 & 2.000000 & 2.000000\end{array}$

$\begin{array}{lll}0.0 & 3.000000 & 2.000000\end{array}$

$\begin{array}{lll}0.0 & 4.000000 & 2.000000\end{array}$

$\begin{array}{lll}0.0 & 5.000000 & 2.000000\end{array}$

$\begin{array}{lll}0.0 & 6.000000 & 2.000000\end{array}$

$\begin{array}{lll}0.0 & 7.000000 & 2.000000\end{array}$

$\begin{array}{lll}0.0 & 7.999999 & 2.000000\end{array}$

$\begin{array}{lll}0.0 & 9.000000 & 2.000000\end{array}$

$0.010 .000000 \quad 2.000000$

$\begin{array}{llll}34 & 0.0 & 0.000000 & 3.000000\end{array}$ 
$0.0 \quad 1.000000 \quad 3.000000$

$0.0 \quad 2.000000 \quad 3.000000$

$0.0 \quad 3.000000 \quad 3.000000$

$0.0 \quad 4.000000 \quad 3.000000$

$0.0 \quad 5.000000 \quad 3.000000$

$0.0 \quad 6.000000 \quad 3.000000$

$0.0 \quad 7.000000 \quad 3.000000$

$0.0 \quad 8.000000 \quad 3.000000$

$0.0 \quad 9.000000 \quad 3.000000$

$0.010 .000000 \quad 3.000000$

$0.0 \quad 0.000000 \quad 4.000000$

$0.0 \quad 1.000000 \quad 4.000000$

$0.0 \quad 2.000000 \quad 4.000000$

$0.0 \quad 3.000000 \quad 4.000000$

$0.0 \quad 4.000000 \quad 4.000000$

$0.0 \quad 5.000000 \quad 4.000000$

$0.0 \quad 6.000000 \quad 4.000000$

$0.0 \quad 7.000001 \quad 4.000000$

$0.0 \quad 8.000000 \quad 4.000000$

$0.0 \quad 9.000000 \quad 4.000000$

$0.0 \quad 10.000000 \quad 4.000000$

$0.0 \quad 0.000000 \quad 5.000000$

$0.0 \quad 1.000000 \quad 5.000000$

$0.0 \quad 2.000000 \quad 5.000000$

$0.0 \quad 3.000000 \quad 5.000000$

$0.0 \quad 4.000000 \quad 5.000000$

$0.0 \quad 5.000000 \quad 5.000000$

$0.0 \quad 6.000000 \quad 5.000000$

$0.0 \quad 7.000000 \quad 5.000000$

$0.0 \quad 8.000000 \quad 5.000000$

$0.0 \quad 9.000000 \quad 5.000000$

$0.010 .000000 \quad 5.000000$

$0.0 \quad 0.000000 \quad 6.000000$

$0.0 \quad 1.000000 \quad 6.000000$

$0.0 \quad 2.000000 \quad 6.000000$

$0.0 \quad 3.000000 \quad 6.000000$

$0.0 \quad 4.000000 \quad 6.000000$

$0.0 \quad 5.000000 \quad 6.000000$

$0.06 .000000 \quad 6.000000$

$0.0 \quad 7.000000 \quad 6.000000$

$0.0 \quad 8.000000 \quad 6.000000$

$0.0 \quad 9.000000 \quad 6.000000$

$0.0 \quad 10.000000 \quad 6.000000$

$0.0 \quad 0.000000 \quad 7.000000$

$0.0 \quad 1.000000 \quad 7.000000$

$\begin{array}{lll}0.0 & 2.000000 & 7.000000\end{array}$

$0.0 \quad 3.000000 \quad 7.000000$

$0.0 \quad 4.000000 \quad 6.999999$

$0.0 \quad 5.000000 \quad 7.000000$

$0.0 \quad 6.000000 \quad 7.000000$

$0.0 \quad 7.000000 \quad 7.000000$

$0.08 .000000 \quad 7.000000$

$\begin{array}{lll}0.0 & 9.000000 \quad 7.000000\end{array}$

$0.010 .000000 \quad 7.000000$

$0.0 \quad 0.000000 \quad 8.000000$

$\begin{array}{lll}0.0 & 1.000000 & 8.000000\end{array}$

$\begin{array}{lll}0.0 & 2.000000 & 7.999999\end{array}$

$0.0 \quad 3.000000 \quad 8.000000$

$0.04 .000000 \quad 8.000000$

$0.0 \quad 5.000000 \quad 8.000000$

$0.0 \quad 6.000000 \quad 8.000000$

$0.0 \quad 7.000000 \quad 8.000000$

$\begin{array}{lll}0.0 & 7.999999 & 7.999999\end{array}$

$0.0 \quad 9.000001 \quad 8.000000$ 


$\begin{array}{rrrr}99 & 0.0 & 10.000000 & 8.000000 \\ 100 & 0.0 & 0.000000 & 9.000000 \\ 101 & 0.0 & 1.000000 & 9.000001 \\ 102 & 0.0 & 2.000000 & 9.000000 \\ 103 & 0.0 & 3.000000 & 9.000000 \\ 104 & 0.0 & 4.000000 & 9.000000 \\ 105 & 0.0 & 5.000000 & 9.000000 \\ 106 & 0.0 & 6.000000 & 9.000000 \\ 107 & 0.0 & 7.000000 & 9.000000 \\ 108 & 0.0 & 8.000000 & 9.000001 \\ 109 & 0.0 & 9.000001 & 9.000001 \\ 110 & 0.0 & 10.000000 & 9.000000 \\ 111 & 0.0 & 0.000000 & 10.000000 \\ 112 & 0.0 & 1.000000 & 10.000000 \\ 113 & 0.0 & 2.000000 & 10.000000 \\ 114 & 0.0 & 3.000000 & 10.000000 \\ 115 & 0.0 & 4.000000 & 10.000000 \\ 116 & 0.0 & 5.000000 & 10.000000 \\ 117 & 0.0 & 6.000000 & 10.000000 \\ 118 & 0.0 & 7.000000 & 10.000000 \\ 119 & 0.0 & 8.000000 & 10.000000 \\ 120 & 0.0 & 9.000000 & 10.000000 \\ 121 & 0.0 & 10.000000 & 10.000000\end{array}$

* element definitions, element number,

* four nodes that make up the element and material number

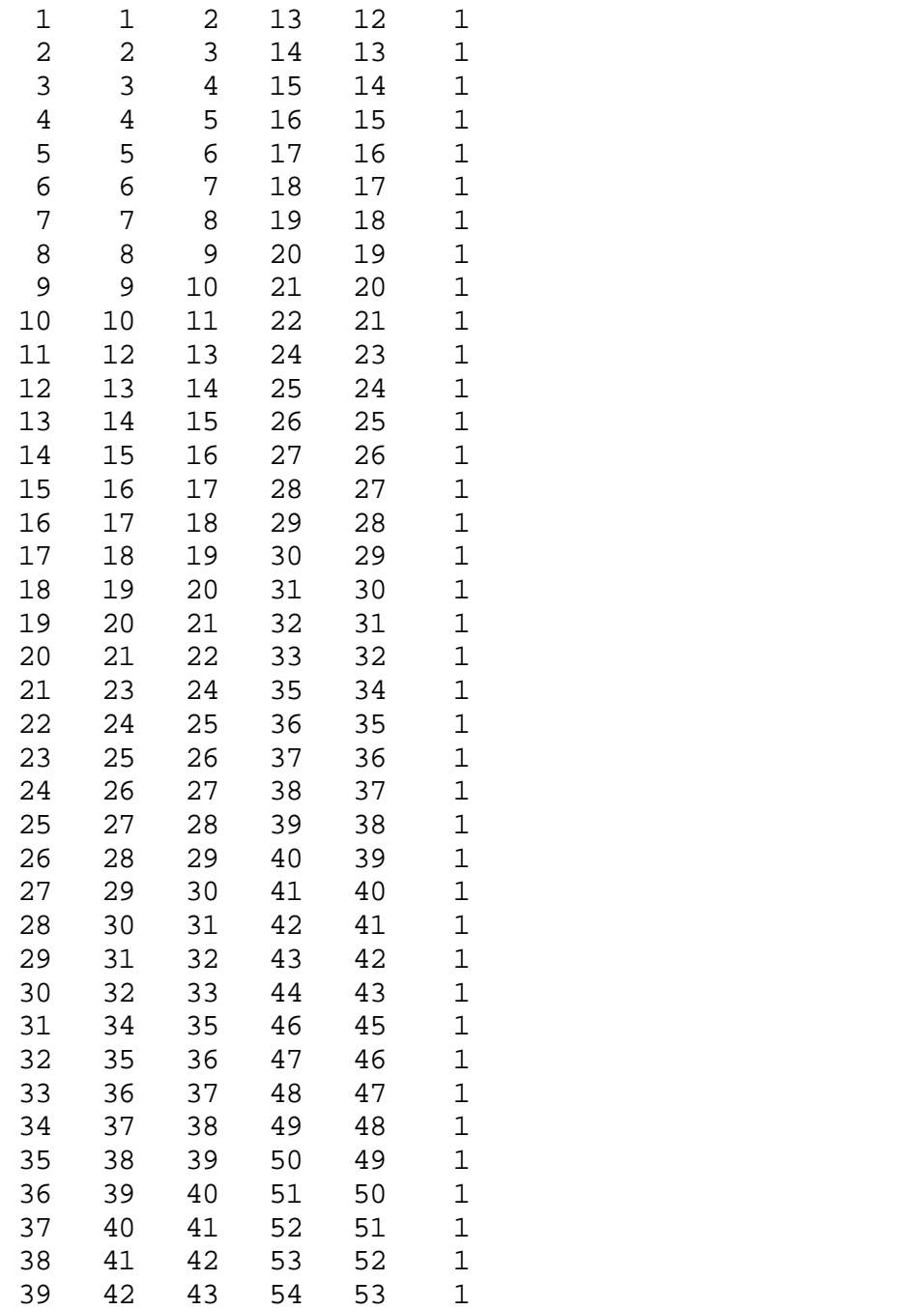




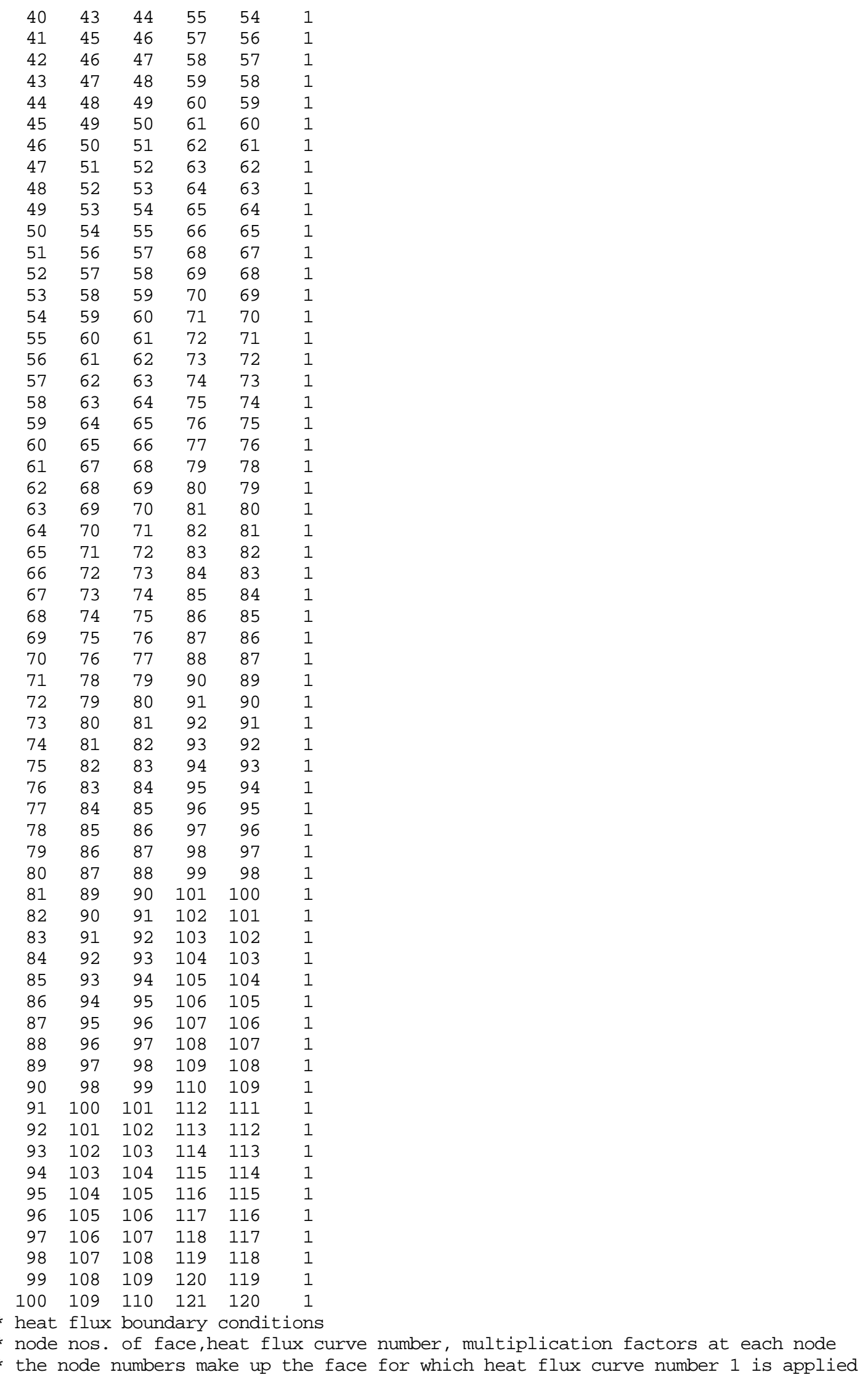




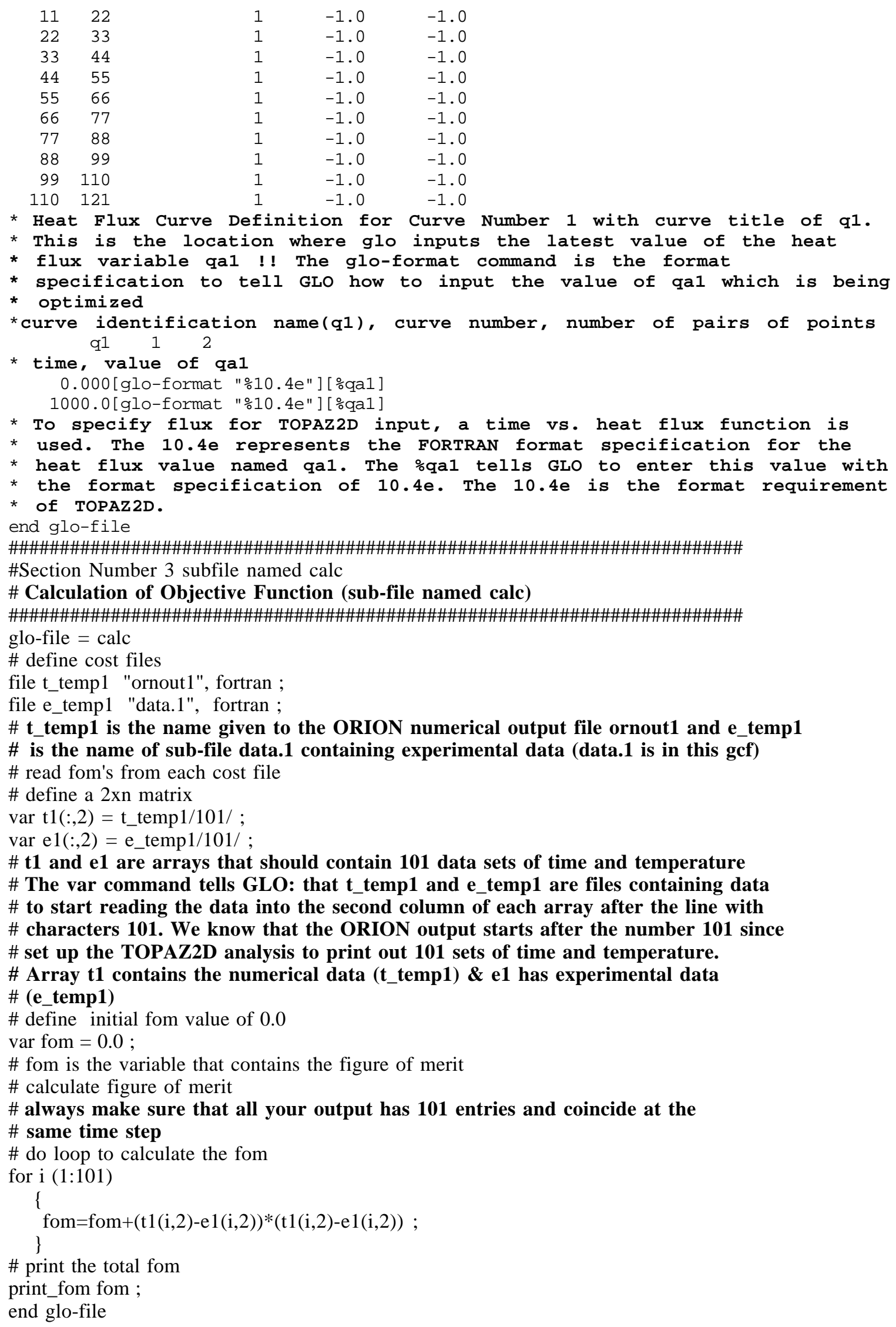




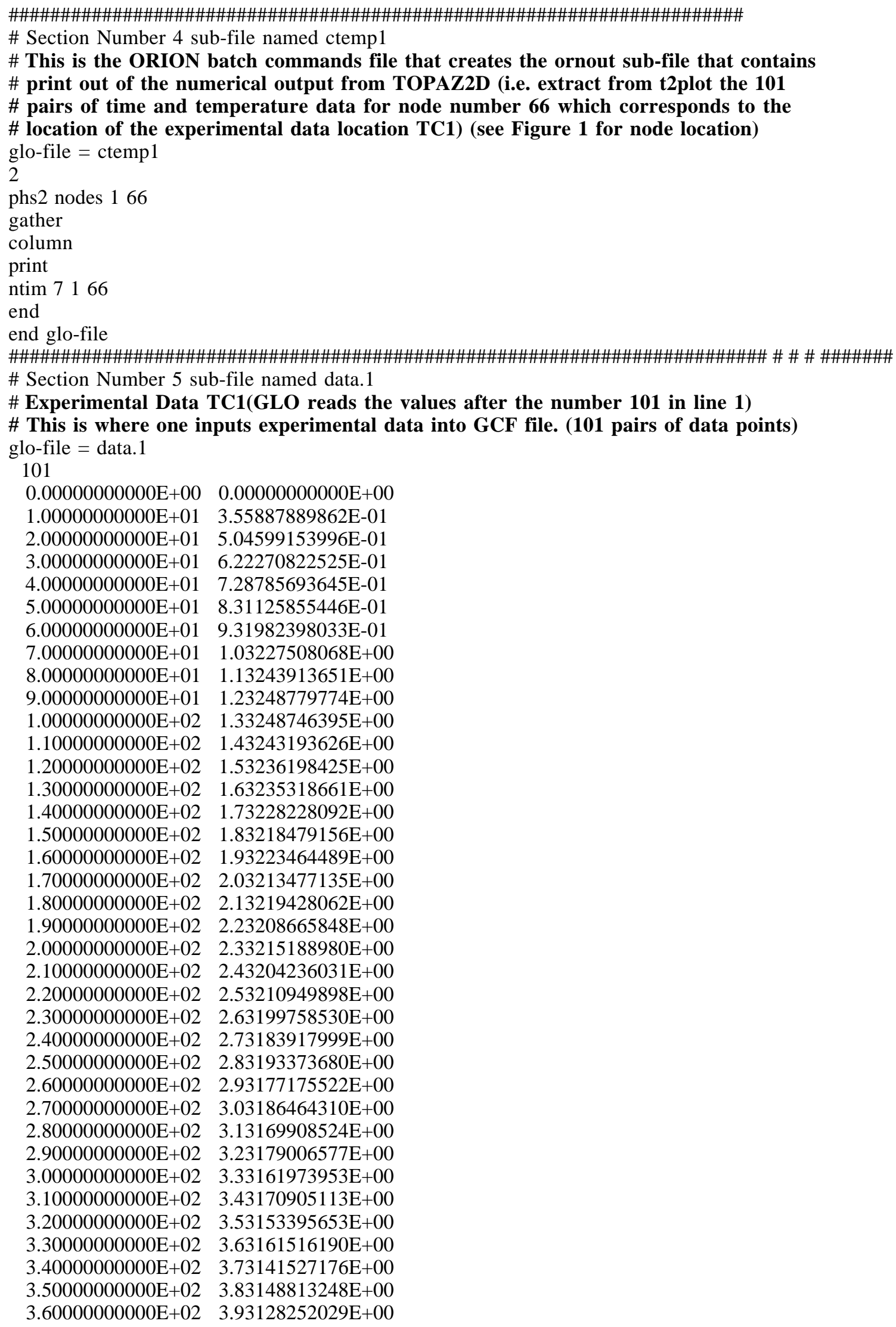


$3.70000000000 \mathrm{E}+02 \quad 4.03135347366 \mathrm{E}+00$

$3.80000000000 \mathrm{E}+02 \quad 4.13114166260 \mathrm{E}+00$

$3.90000000000 \mathrm{E}+02 \quad 4.23121070862 \mathrm{E}+00$

4.00000000000E+02 4.33099317551E+00

$4.10000000000 \mathrm{E}+02 \quad 4.43106031418 \mathrm{E}+00$

$4.20000000000 \mathrm{E}+02 \quad 4.53083658218 \mathrm{E}+00$

$4.30000000000 \mathrm{E}+02 \quad 4.63090276718 \mathrm{E}+00$

$4.40000000000 \mathrm{E}+02 \quad 4.73067235947 \mathrm{E}+00$

$4.50000000000 \mathrm{E}+02 \quad 4.83073759079 \mathrm{E}+00$

$4.60000000000 \mathrm{E}+02 \quad 4.93047666550 \mathrm{E}+00$

$4.70000000000 \mathrm{E}+02 \quad 5.03014802933 \mathrm{E}+00$

$4.80000000000 \mathrm{E}+02 \quad 5.13023948669 \mathrm{E}+00$

$4.90000000000 \mathrm{E}+02 \quad 5.22990655899 \mathrm{E}+00$

$5.00000000000 \mathrm{E}+02 \quad 5.32999753952 \mathrm{E}+00$

$5.10000000000 \mathrm{E}+02 \quad 5.42966079712 \mathrm{E}+00$

$5.20000000000 \mathrm{E}+02 \quad 5.52975082397 \mathrm{E}+00$

$5.30000000000 \mathrm{E}+02 \quad 5.62941026688 \mathrm{E}+00$

$5.40000000000 \mathrm{E}+02 \quad 5.72949981689 \mathrm{E}+00$

$5.50000000000 \mathrm{E}+02 \quad 5.82915592194 \mathrm{E}+00$

$5.60000000000 \mathrm{E}+02 \quad 5.92924499512 \mathrm{E}+00$

$5.70000000000 \mathrm{E}+02 \quad 6.02884578705 \mathrm{E}+00$

$5.80000000000 \mathrm{E}+02 \quad 6.12889862061 \mathrm{E}+00$

$5.90000000000 \mathrm{E}+02 \quad 6.22850465775 \mathrm{E}+00$

$6.00000000000 \mathrm{E}+02 \quad 6.32855701447 \mathrm{E}+00$

$6.10000000000 \mathrm{E}+02 \quad 6.42816686630 \mathrm{E}+00$

$6.20000000000 \mathrm{E}+02 \quad 6.52821969986 \mathrm{E}+00$

$6.30000000000 \mathrm{E}+02 \quad 6.62783336639 \mathrm{E}+00$

$6.40000000000 \mathrm{E}+02 \quad 6.72735404968 \mathrm{E}+00$

$6.50000000000 \mathrm{E}+02 \quad 6.82743597031 \mathrm{E}+00$

$6.60000000000 \mathrm{E}+02 \quad 6.92692661285 \mathrm{E}+00$

$6.70000000000 \mathrm{E}+02 \quad 7.02700901031 \mathrm{E}+00$

$6.80000000000 \mathrm{E}+02 \quad 7.12651538849 \mathrm{E}+00$

$6.90000000000 \mathrm{E}+02 \quad 7.22659826279 \mathrm{E}+00$

$7.00000000000 \mathrm{E}+02 \quad 7.32611751556 \mathrm{E}+00$

$7.10000000000 \mathrm{E}+02 \quad 7.42614650726 \mathrm{E}+00$

$7.20000000000 \mathrm{E}+02 \quad 7.52560758591 \mathrm{E}+00$

$7.30000000000 \mathrm{E}+02 \quad 7.62564086914 \mathrm{E}+00$

$7.40000000000 \mathrm{E}+02 \quad 7.72512865067 \mathrm{E}+00$

$7.50000000000 \mathrm{E}+02 \quad 7.82516574860 \mathrm{E}+00$

$7.60000000000 \mathrm{E}+02 \quad 7.92464399338 \mathrm{E}+00$

$7.70000000000 \mathrm{E}+02 \quad 8.02400398254 \mathrm{E}+00$

$7.80000000000 \mathrm{E}+02 \quad 8.12407588959 \mathrm{E}+00$

$7.90000000000 \mathrm{E}+02 \quad 8.22347640991 \mathrm{E}+00$

$8.00000000000 \mathrm{E}+02 \quad 8.32355117798 \mathrm{E}+00$

$8.10000000000 \mathrm{E}+02 \quad 8.42288780212 \mathrm{E}+00$

$8.20000000000 \mathrm{E}+02 \quad 8.52290248871 \mathrm{E}+00$

$8.30000000000 \mathrm{E}+02 \quad 8.62229347229 \mathrm{E}+00$

$8.40000000000 \mathrm{E}+02 \quad 8.72158527374 \mathrm{E}+00$

$8.50000000000 \mathrm{E}+02 \quad 8.82164192200 \mathrm{E}+00$

$8.60000000000 \mathrm{E}+02 \quad 8.92093086243 \mathrm{E}+00$

$8.70000000000 \mathrm{E}+02 \quad 9.02099514008 \mathrm{E}+00$

$8.80000000000 \mathrm{E}+02 \quad 9.12030315399 \mathrm{E}+00$

$8.90000000000 \mathrm{E}+02 \quad 9.22029685974 \mathrm{E}+00$

$9.00000000000 \mathrm{E}+02 \quad 9.31960296631 \mathrm{E}+00$

$9.10000000000 \mathrm{E}+02 \quad 9.41881561279 \mathrm{E}+00$

$9.20000000000 \mathrm{E}+02 \quad 9.51886081696 \mathrm{E}+00$

$9.30000000000 \mathrm{E}+02 \quad 9.61808586121 \mathrm{E}+00$

$9.40000000000 \mathrm{E}+02 \quad 9.71814346313 \mathrm{E}+00$ 


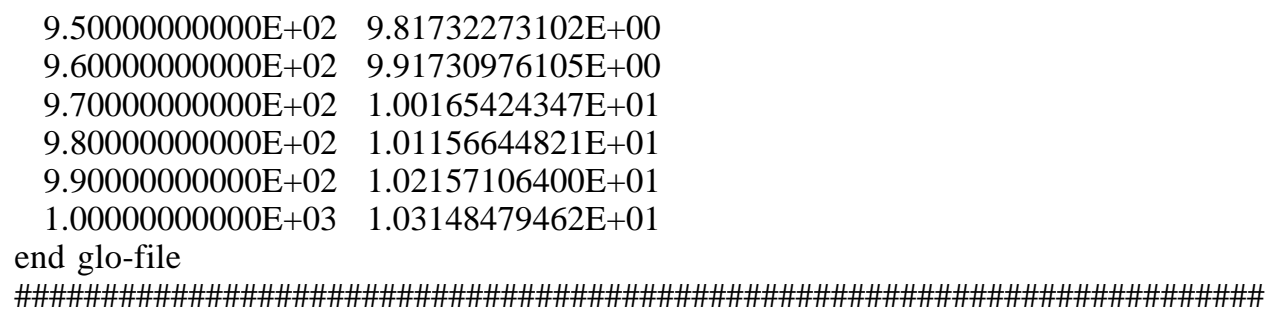

\section{Appendix. Results Files}

A. solution.0000 file (gives number of cyles, final value of qa1, and fom)

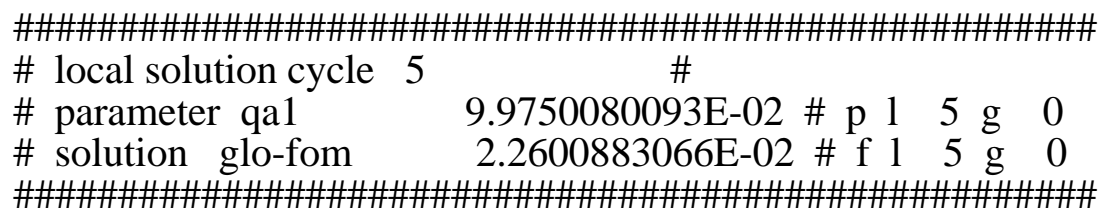

B. local.u2 file (gives the value of the fom for each iteration)

$$
\begin{array}{cl}
\text { \# fom } \\
1 & 5.7858497519 \mathrm{E}+04 \\
2 & 5.8003234021 \mathrm{E}+04 \\
3 & 3.6089273876 \mathrm{E}+03 \\
4 & 3.5728923923 \mathrm{E}+03 \\
5 & 2.2600883066 \mathrm{E}-02
\end{array}
$$

C. local.I1 file (for each iteration, it reproduces the commands done in each iteration, lists the value of the figure of merit, the value of qal used in each iteration and the type of iteration [initial run, newton step, gradient evaluation, etc.])

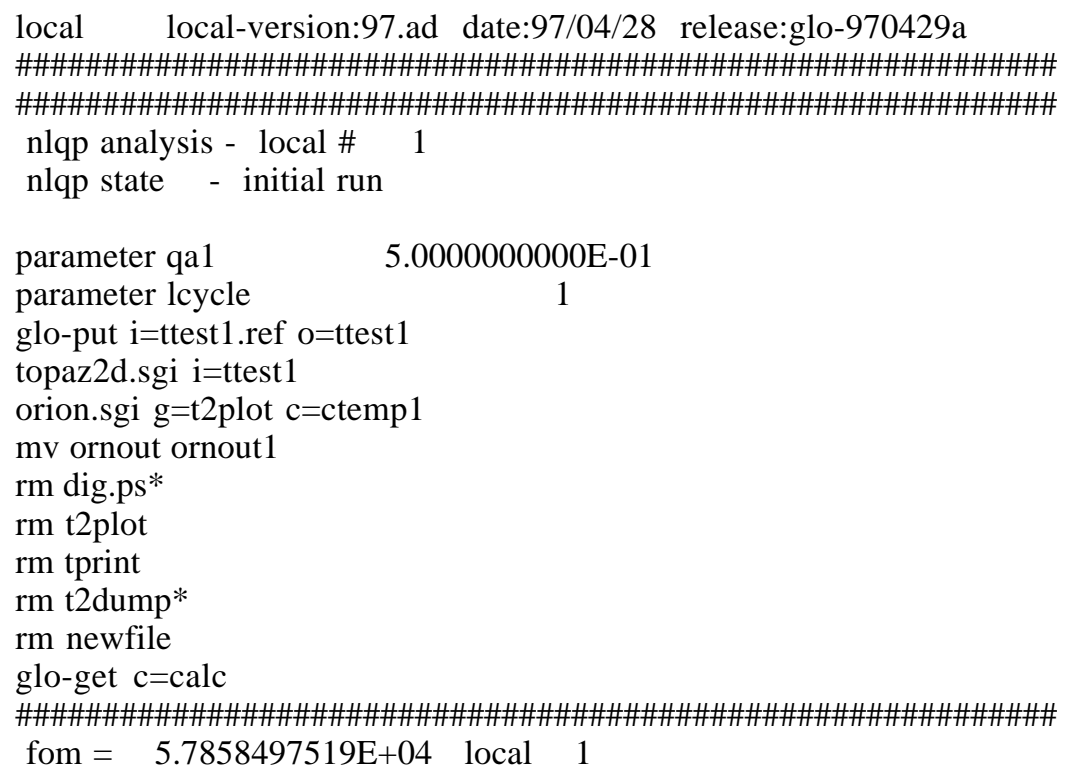




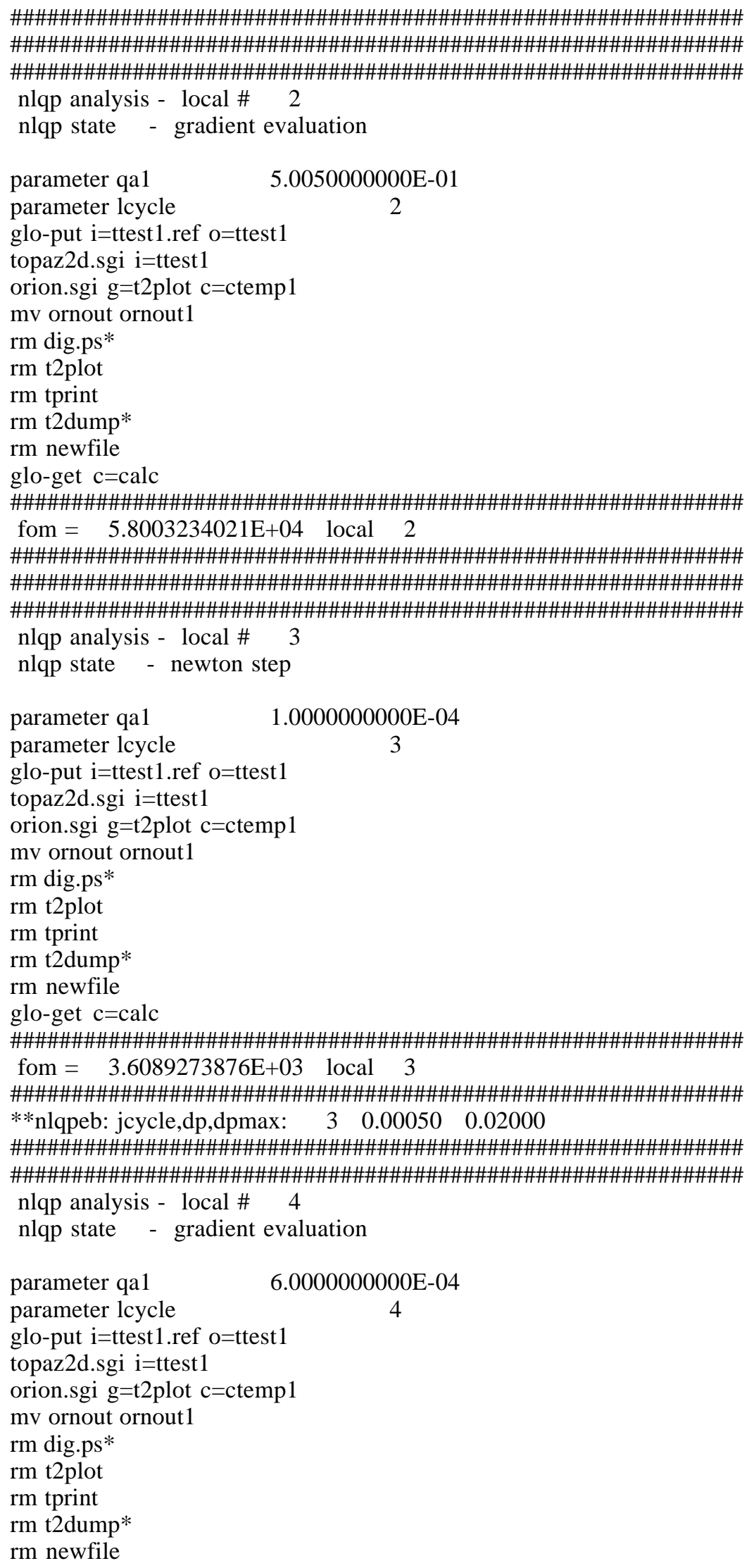


glo-get $\mathrm{c}=$ calc

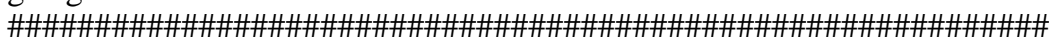

fom $=3.5728923923 \mathrm{E}+03$ local 4

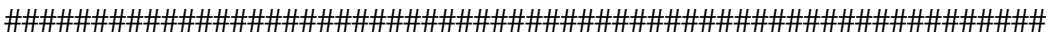

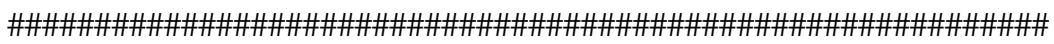

nlqp analysis - local \# 5

nlqp state - newton step

parameter qa1 $\quad 9.9750080093 \mathrm{E}-02$

parameter lcycle

5

glo-put $\mathrm{i}=$ ttest 1 .ref $\mathrm{o}=$ ttest 1

topaz2d.sgi $\mathrm{i}=$ ttest 1

orion.sgi $\mathrm{g}=\mathrm{t} 2$ plot $\mathrm{c}=$ ctemp1

mv ornout ornout1

rm dig.ps*

rm t2plot

rm tprint

rm t2dump*

rm newfile

glo-get $c=$ calc

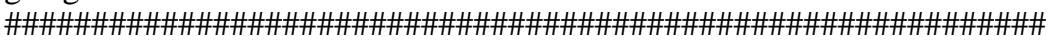

fom $=2.2600883066 \mathrm{E}-02$ local 5

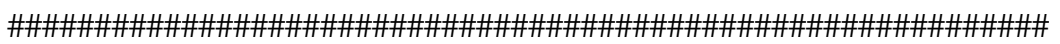

**nlqpeb: jcycle,dp,dpmax: $50.00010 \quad 0.02000$

D. local.12 file (gives in a summarized format some of the information given in local.12 i.e. value of the figure of merit, the value of qa1 used in each iteration and the glo state)

local local-version:97.ad date:97/04/28 release:glo-970429a

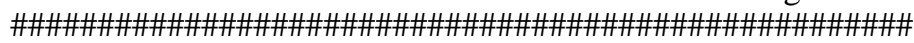

\# solution glo-run local 1 \#

\# glo state initial run

\# parameter qa1

\#

\# solution glo-fom $\quad 5.7858497519 \mathrm{E}+04$ \# f 11

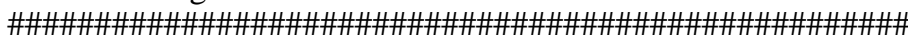

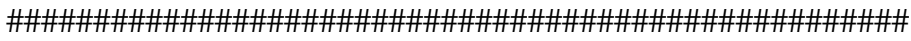

\# solution glo-run local 2 \#

\# glo state gradient evaluation \#

\# parameter qa1 5.0050000000E-01 \# p 1

\# solution glo-fom $\quad 5.8003234021 E+04$ \# $\mathrm{f} 1$

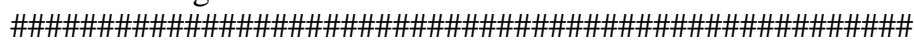

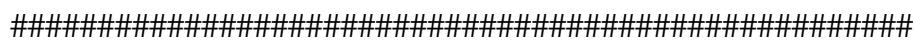

\# solution glo-run local 3 \#

\# glo state newton step \#

\# parameter qa1 $1.0000000000 \mathrm{E}-04$ \# p 13

\# solution glo-fom $3.6089273876 E+03$ \# $\mathrm{f}$ l 3

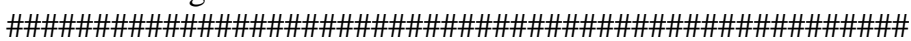

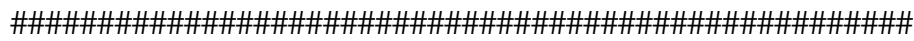

\# solution glo-run local 4 \#

\# glo state gradient evaluation \#

\# parameter qa1 6.0000000000E-04 \# p 14

\# solution glo-fom $3.5728923923 \mathrm{E}+03$ \# $\mathrm{f}$ l 4

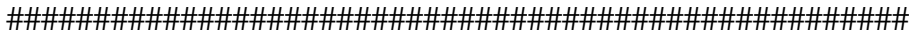

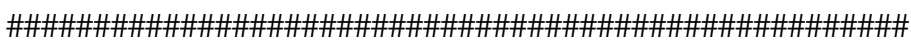

\# solution glo-run local 5 \#

\# glo state newton step

\# parameter qa1

\#

\# solution glo-fom

9.9750080093E-02 \# p 15

$2.2600883066 \mathrm{E}-02$ \# f 15 


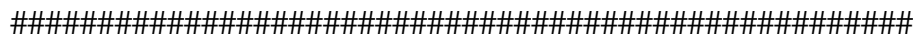

